Check for updates

Cite this: Chem. Sci., 2019, 10, 4896

๑ All publication charges for this article have been paid for by the Royal Society of Chemistry

Received 13th February 2019

Accepted 25th March 2019

DOI: $10.1039 / \mathrm{c} 9 \mathrm{sc00757a}$

rsc.li/chemical-science

\section{Conformer-dependent self-assembled metallacycles with photo-reversible response $\uparrow$}

\author{
Mengqi Li, ț ${ }^{\mathrm{a}}$ Li-Jun Chen,,$_{+}^{\mathrm{b}}$ Zhipeng Zhang, ${ }^{\mathrm{a}}$ Qianfu Luo, ${ }^{a}$ Hai-Bo Yang, (D) *b \\ He Tian ${ }^{a}$ and Wei-Hong Zhu (DD *a
}

Discrete, well-defined metallacycles and metallacages with stimuli-responsive behaviors have been largely predominated by the organic donor/metal acceptor paradigm with spontaneous formation of coordination bonds. However, light-driven self-assembly systems usually show relatively low utilization yield of photons and low fatigue resistance. Given that almost no example illustrates the different self-assembly behaviors of antiparallel and parallel conformers in the traditional photochromic diarylethene (DAE) system, here we have for the first time constructed a unique series of photoactive conformer-dependent metallacycles, focusing on the characterization and comparison of self-assembly behavior in different ligand conformers with different di-platinum(II) acceptors. Their photoswitchable scaffold sizes and shapes are precisely controlled by photochromically separable parallel or anti-parallel conformers via coordinationdriven self-assembly. The ap-conformer and closed form provide larger bending angles upon coordination with di-Pt(I) acceptors into hexagon [6+6] or [3+3] while the $p$-conformer only can form smaller polygon cycles. Notably, in contrast with the non-photoactive parallel conformer, the reversible interconversion of anti-parallel ring-open and ring-closed conformer metallacycles can be achieved by alternate irradiation with UV and visible light, respectively, along with a relatively high conversion ratio and good fatigue resistance. This work provides a potential way to construct smart materials for use in sensing, catalysis and drug delivery systems.

\section{Introduction}

Coordination-driven self-assembly has provided a bottom-up basis for mimicking stimuli-responsive behaviors in artificial systems. ${ }^{1-13}$ Among external stimuli such as $\mathrm{pH}$, redox potential and magnetic field, ${ }^{\mathbf{1 4 - 2 1}}$ light is well considered as a perfect external trigger to control structural transformations in supramolecular entities since photon input is a clean and precise spatiotemporal control in a remote and nondestructive manner. ${ }^{22-28}$ Among the most ideal candidates, photochromic diarylethene (DAE) derivatives ${ }^{29-39}$ can undergo reversible transformation between open- and closed-ring states, along with light-induced changes in their optical and electronic

${ }^{a}$ Key Laboratory for Advanced Materials, Institute of Fine Chemicals, Shanghai Key Laboratory of Functional Materials Chemistry, Joint International Research Laboratory of Precision Chemistry and Molecular Engineering, Feringa Nobel Prize Scientist Joint Research Center, School of Chemistry and Molecular Engineering, East China University of Science and Technology, Shanghai 200237, China. E-mail: whzhu@ecust.edu.cn

${ }^{b}$ Shanghai Key Laboratory of Green Chemistry and Chemical Processes, Chang-Kung Chuang Institute, School of Chemistry and Molecular Engineering, East China Normal University, Shanghai 200062,China.E-mail: hbyang@chem.ecnu.edu.cn

$\dagger$ Electronic supplementary information (ESI) available. CCDC 1848524. For ESI and crystallographic data in CIF or other electronic format see DOI: 10.1039/c9sc00757a

\$ These authors contributed equally to this work. properties. Recent efforts have been devoted to constructing light-driven supramolecular systems such as light-controlled interconversion between a self-assembled triangle and a rhombicuboctahedral sphere and uptake or release of anionic guests using a photochromic coordination cage. ${ }^{\mathbf{4 0 , 4 1}}$ Recently, we have also achieved the construction of multibisthienylethene hexagons with photoreversible supramolecule-to-supramolecule interchanges between the specific photochromic ring-open and ring-closed states. ${ }^{23}$ However, due to the low-efficiency separation of anti-parallel and parallel conformers, DAE-based self-assemblies usually show relatively low quantum yield of photons. Meanwhile, a rare example has illustrated the different self-assembly behavior of anti-parallel and parallel conformers. ${ }^{40}$ The mixed configuration of anti-parallel and parallel conformers usually brings complexity and difficulties to the research. Understanding the different self-assembly behavior would also contribute to the construction of various supramolecular systems based on diarylethenes.

Incorporation of the sterically hindered bisbenzo(thiadiazole) ethene bridge by our group can completely block the conformer interchange, ${ }^{\mathbf{4 2 - 4 5}}$ the two conformers can be easily separated using a column chromatograph, and the resulting anti-parallel conformer exhibits high photocyclization quantum yields $\left(\Phi_{\mathrm{o}-\mathrm{c}}\right)$ as well as excellent thermal stability. With this in mind, here we report a unique series of conformer-dependent 
metallacycles based on sterically hindered ethene bridge diarylethenes as donor building blocks, resulting from selfassembly with different di-platinum(II) ligand acceptors. As a consequence, unusual discrete, well-defined supramolecular multidithienylethene metallacycles were established with several characteristics: (i) the conformer-dependent photoswitchable scaffold sizes and shapes precisely modulated by photochromic parallel or anti-parallel conformers via coordination-driven self-assembly, (ii) the uncommon conformer-based self-sorting supramolecular behavior, and (iii) the reversible transformation between anti-parallel and ringclosed conformers with a relatively high conversion ratio and good fatigue resistance via alternate irradiation with UV and visible light. We focused on characterizing the self-assembly behavior between different photoresponsive ligand conformers with different di-Pt(II) acceptors, particularly for comparing the photo-reversible response of self-assembled metallacycles.

\section{Results and discussion}

\section{Conformer separation of photoswitchable ligands}

For the traditional photochromic DAE series, the open form can be divided into two conformers: anti-parallel (ap-) configuration with $C_{2}$ symmetry and parallel (p-) configuration with mirror symmetry. According to the Woodward-Hoffmann rule, only the ap-conformer can undergo photocyclization while the pconformer cannot. Due to the typical rapid rotation of flexible aryl groups, two conformers cannot be separated efficiently in the typical photochromic DAE system. In fact, most DAE derivatives show relatively low photocyclization quantum yields which limits their practical device application. ${ }^{46-49}$ To solve the problem, our group has recently introduced a sterically hindered bisbenzo(thiadiazole) ethene bridge and large bulky benzothiophene unit to limit the rotation of the aryl group, even successfully separating ap- and p-conformers. ${ }^{42}$ Generally, lightcontrolled self-assemblies usually show relatively low fatigue resistance and quite low utilization yield of photons. ${ }^{19,50,51}$ For common DAE series, the mixture of ap- and p-conformers also limits the photoresponse efficiency. ${ }^{40}$ Hence, separation of two conformers of the DAE series by introducing a sterically hindered ethene bridge can be expected to make a breakthrough to the limitation.

For developing photo-induced metallacycles via selfassembly behavior, we borrowed the sterically hindered ethene bridge DAE concept to develop a photoactive organic ligand donor (PY, Scheme 1). That is, we incorporated a bulky aryl group (pyridine-substituted benzothiophene) into the hindered ethene bridge of bisbenzo(thiadiazole). The target photoswitchable molecule PY was synthesized via Suzuki coupling between the corresponding dibromo substituted intermediate and pyridine borate. Taken together with the large bulky effect, the rotation between the ethene bridge and the side chain group is completely blocked due to the high rotation barrier, thus leading to the successful separation of ap- and pconformers (ap-PY and p-PY, Scheme 1) by common silica gel

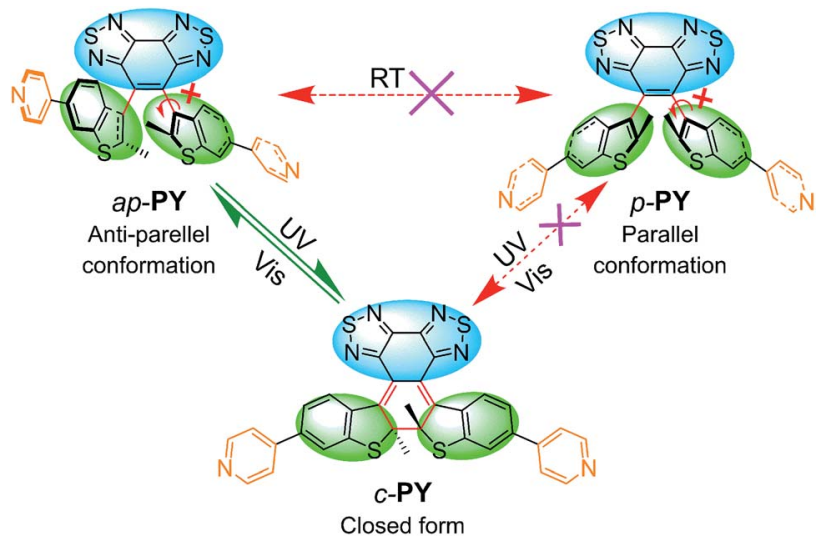

Scheme 1 Chemical structures and isomerism of PY. The conversion relationship of ap-PY, $\mathrm{p}-\mathrm{PY}$ and c-PY with a sterically hindered bisbenzo(thiadiazole) ethene bridge (blue unit) and bulky aryl group (green unit). Note: "ap" and " $\mathrm{p}$ " represent the anti-parallel and parallel conformation, respectively, while " $c$ " represents the closed form. Two open forms are thermally isolated (red dashed arrows) due to blocked isomerization of high rotation barrier. The parallel ring open isomer is photochemically inert (red dashed arrows), and only the anti-parallel conformer is able to reversibly transform to the closed form (olive arrows), according to the Woodward-Hoffmann rule on electrochemical cyclization of a $4 n+2 \pi$-electron system.

chromatography. The closed form c-PY was prepared by UV irradiation of ap-PY. ${ }^{44}$

\section{Self-assembly of parallel conformers into triangle and rhombus metallacycles}

Due to the different conformation of these isomers, it is attractive enough to study the difference of self-assembly behavior with the three separated conformers ap-PY, p-PY and c-PY. According to the "directional bonding" model and the "symmetry interaction" model, ${ }^{1,52}$ the final geometry of the resulting individual two-dimensional polygons is dependent upon the value of turning angle with their angular components. That is, the shape and size of metallacycles have a critical effect upon different bending angles of photoresponsive conformer ligands and di-platinum acceptors. As a result of the successful separation of ap-PY, p-PY and c-PY, we have an opportunity to compare the self-assembly difference between ap- and $\mathrm{p}$ conformers.

Our previous study ${ }^{23}$ showed that for traditional diarylethenes, compared to parallel conformers, anti-parallel is the preferable conformation to self-assemble with di-platinum acceptors into a two-dimensional polygon. Here, given that we established successful separation of ap-PY and p-PY, we have an opportunity to study the self-assembly behavior of parallel conformers.

First, we focused on the self-assembly of p-PY with the diplatinum ligand di-Pt(II) 1 (Scheme 2). The triangle $\mathrm{p}-[3+3]$ can be easily obtained by stirring mixtures of p-PY with $180^{\circ}$ di$\mathrm{Pt}(\mathrm{II})$ acceptor 1 in $1: 1$ molar ratio in $\mathrm{CD}_{2} \mathrm{Cl}_{2}$ (Scheme $\mathrm{S} 1 \dagger$ ). The quantitative self-assembly formation of a discrete triangle of $\mathrm{p}$ $[3+3]$ was confirmed through multinuclear NMR $\left({ }^{1} \mathrm{H}\right.$ and $\left.{ }^{31} \mathrm{P}\right)$, 


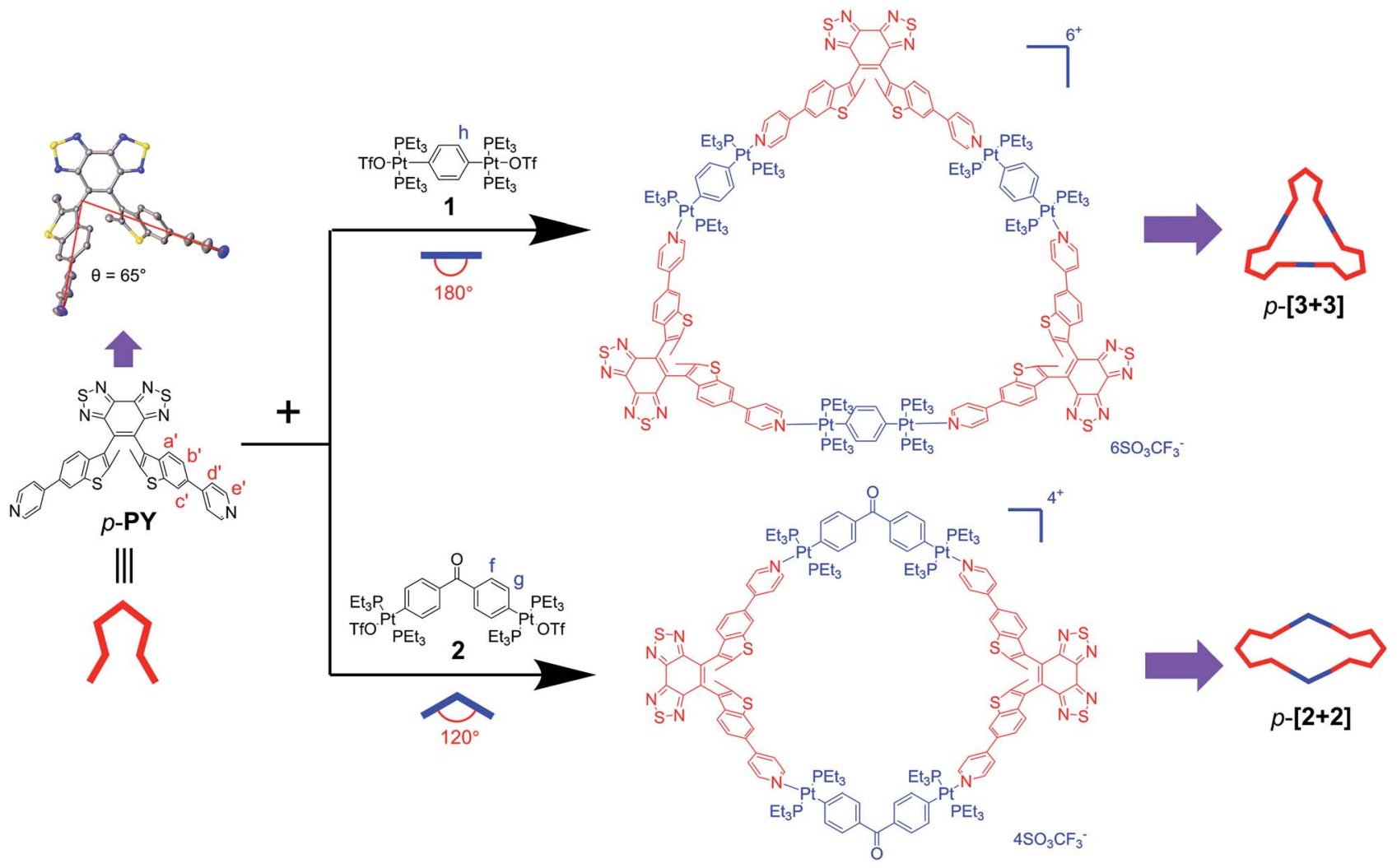

Scheme 2 Graphical representation of self-assembly of $\mathrm{p}$-PY with $180^{\circ}$ and $120^{\circ}$ acceptors 1 and 2 with ORTEP representation of X-ray single crystal structures of $\mathrm{p}-\mathrm{PY}$ drawn with $50 \%$ probability. The red line shows the bending angle of two pyridines.

showing a single discrete assembly with high symmetry (Fig. 1). In the simple ${ }^{1} \mathrm{H}$ NMR pattern of p-[3+3], the pyridine protons showed a significant downfield shift (ca. 0.15 and $0.49 \mathrm{ppm}$ for $H_{\mathrm{e}^{\prime}}$ and $H_{\mathrm{d}^{\prime}}$ respectively) with respect to those of p-PY due to the loss of electron density upon complexation of the pyridine $N$ atom with the $\mathrm{Pt}$ (II) metal center. At the same time, the protons in the benzothiophene unit also exhibited a downfield shift for $H_{\mathrm{a}^{\prime}}, H_{\mathrm{b}^{\prime}}$ and $H_{\mathrm{c}^{\prime}}$, respectively (Fig. 1a). Moreover, the ${ }^{31} \mathrm{P}$ NMR spectrum clearly displayed a single peak indicating a high symmetry structure of $\mathrm{p}-[3+3]$. Meanwhile, an upfield shift was found from the corresponding starting platinum acceptor to metallacycles by $c a$. $8.05 \mathrm{ppm}$ for $\mathrm{p}-[3+3]$, which can be ascribed to the increase in the electron density of the $\mathrm{P}$ atom upon coordination. This change as well as the decrease in the coupling of flanking ${ }^{195} \mathrm{Pt}$ satellites $(\Delta J=152.5 \mathrm{~Hz})$ is consistent with the electron back-donation from Pt (Fig. 1b). Here the well- a)

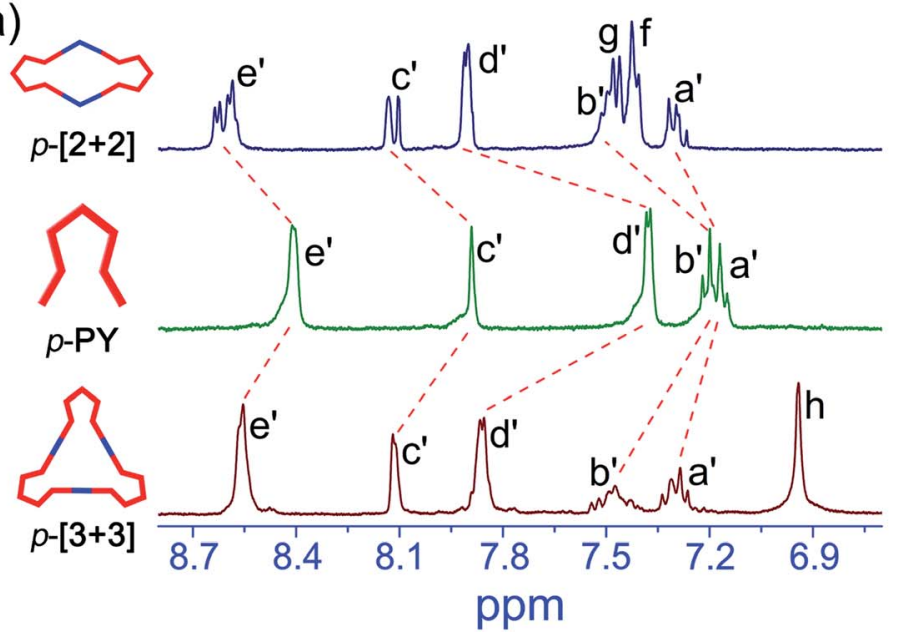

b)

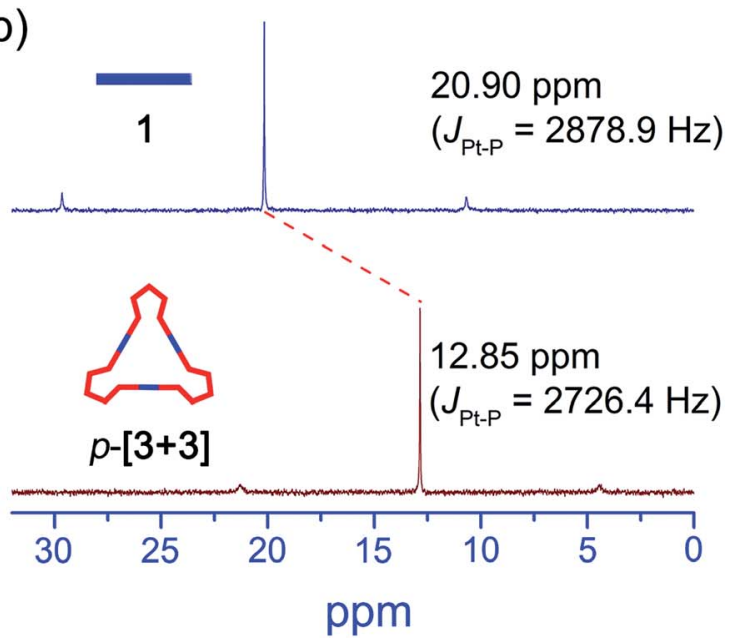

Fig. 1 Characterization of $p-[3+3]$ and $p-[2+2]$. (a) Partial ${ }^{1} \mathrm{H}$ NMR spectra $(400 \mathrm{MHz}, 293 \mathrm{~K})$ of $p-[2+2], p-P Y$ and $p-[3+3]$ in $C D_{2} C l_{2}$. (b) ${ }^{31} \mathrm{P}$ NMR spectra (161.9 MHz, $\mathrm{CD}_{2} \mathrm{Cl}_{2}, 293 \mathrm{~K}$ ) of $180^{\circ}$ di-Pt(॥) acceptor 1 and $\mathrm{p}-[3+3]$. 
defined signals of both ${ }^{1} \mathrm{H}$ and ${ }^{31} \mathrm{P}$ NMR fully support the formation of a perfect structure as a sole assembly product.

The stoichiometry in formation of discrete triangles can be investigated by electrospray ionization time of flight mass spectrometry (ESI-TOF-MS), providing further support to the existence of $\mathrm{p}$-[3 +3$]$. The ESI-TOF-MS spectrum of $\mathrm{p}-[3+3]$ (Fig. 2a) shows peaks at $m / z=1728.11,1258.36$, and 977.50 (Fig. 2a; S1 and S2 in the ESI $\dagger$ ), exactly corresponding to [M3OTf $]^{3+},[\mathrm{M}-4 \mathrm{OTf}]^{4+}$ and $[\mathrm{M}-5 \mathrm{OTf}]^{5+}$. The peaks were isotopically resolved and agreed very well with their calculated theoretical distribution of the assigned [3 +3$]$ assembly.

Then we further studied the self-assembly of p-PY with smaller angle $\left(120^{\circ}\right)$ di-Pt(II) acceptor 2 , which could produce smaller metallacycles. A rhombus $\mathrm{p}-[2+2]$ with a smaller size was obtained with the quantitative self-assembly of p-PY with $120^{\circ}$ di-Pt(II) acceptor 2 (Scheme S1†). The quantitative selfassembly formation of rhombus $\mathrm{p}-[2+2]$ was confirmed by multinuclear NMR $\left({ }^{1} \mathrm{H}\right.$ and $\left.{ }^{31} \mathrm{P}\right)$ spectroscopy (Fig. 1a and $\left.\mathrm{S} 3 \dagger\right)$. The ${ }^{1} \mathrm{H}$ NMR spectra indicated a similar downfield shift in both pyridine and benzothiophene. The ${ }^{31} \mathrm{P}$ NMR spectra also showed an upfield shift from initial di-platinum acceptor 2, along with a decrease in the coupling of flanking ${ }^{195} \mathrm{Pt}$ satellites $(\Delta J=132.5 \mathrm{~Hz}$, Fig. S3†). However, there was an unexpected phenomenon in the ${ }^{1} \mathrm{H}$ and ${ }^{31} \mathrm{P}$ NMR spectra of $\mathrm{p}-[2+2]$ compared with those of $\mathrm{p}-[3+3]$. In the ${ }^{1} \mathrm{H}$ NMR spectrum of $\mathrm{p}$ [2+2], the $H_{\mathrm{e}^{\prime}}$ of pyridines showed two doublets near $8.61 \mathrm{ppm}$ while $H_{\mathrm{c}^{\prime}}$ of benzothiophene showed two singlets at about $8.12 \mathrm{ppm}$. Moreover, the signal of the phosphine group in the

${ }^{31} \mathrm{P}$ NMR spectrum also displayed two very close singlets. Considering the hindered rotation of the Pt-N (pyridine) bond, this would also be attributed to the hindered rotation between

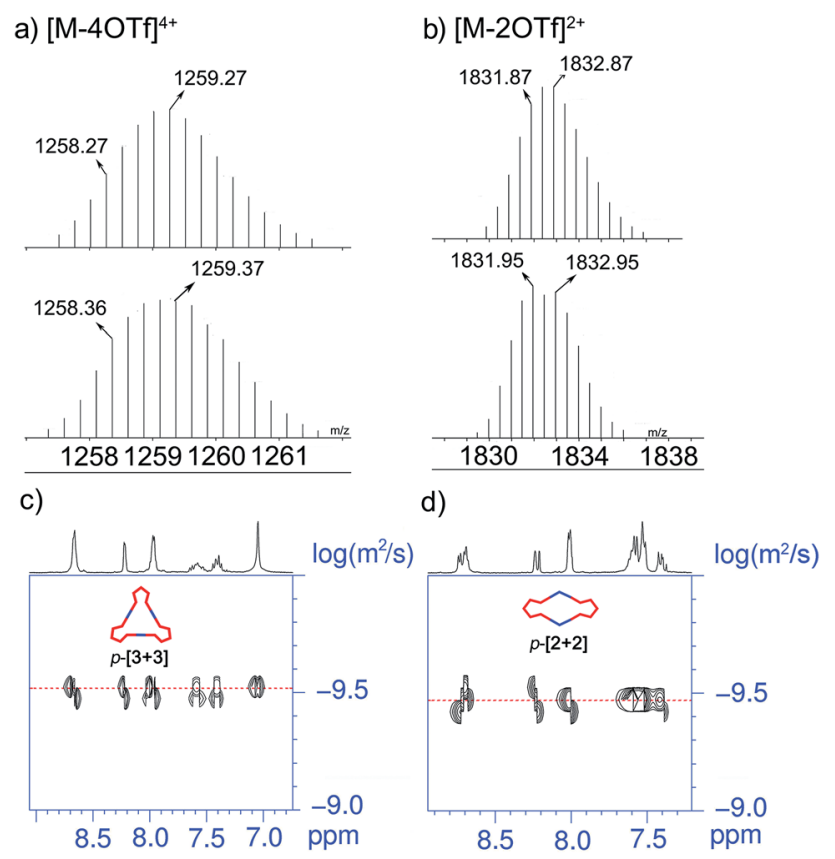

Fig. 2 Theoretical (top) and experimental (bottom) ESI-TOF-MS results of $p-[3+3]$ of (a) $[\mathrm{M}-4 \mathrm{OTf}]^{4+}$ and $p-[2+2]$ of (b) $[\mathrm{M}-2 \mathrm{OTf}]^{2+}$. Partial 2D DOSY spectra of (c) p-[3 + 3] and (d) p-[2+2] $(400 \mathrm{MHz}$, $\left.\mathrm{CD}_{2} \mathrm{Cl}_{2}, 293 \mathrm{~K}\right)$. the bisbenzo(thiadiazole) ethene bridge and benzothiophene units, thus resulting in large ring tension induced by small cycles for protons of pyridine and benzothiophene units. ${ }^{53-55}$ Different from $\mathrm{p}-[2+2], \mathrm{p}-[3+3]$ possesses a larger size of metallacycles which reduces the steric hindrance between the ethene bridge and the benzothiophene unit.

Interestingly, upon changing the di-Pt(II) acceptor from $\mathbf{1}$ $\left(180^{\circ}\right)$ to $2\left(120^{\circ}\right)$, we have successfully obtained discrete metallacycles with different sizes and shapes, which can be attributed to the different bending angles of di-Pt(II) acceptors. Two dimensional (2D) diffusion-ordered ${ }^{1} \mathrm{H}$ NMR spectroscopy (DOSY) was applied as an advanced technique to measure the size of the supramolecular system in solution. The observation of a single band at $\log D=-9.45$ and -9.50 also confirmed the formation of a single self-assembled product (Fig. 2c and d). Meanwhile, the diffusion coefficient of $\mathrm{p}-[3+3]\left(3.16 \times 10^{-10}\right.$ $\left.\mathrm{m}^{2} \mathrm{~s}^{-1}\right)$ is obviously smaller than that of $\mathrm{p}-[2+2]\left(3.55 \times 10^{-10}\right.$ $\left.\mathrm{m}^{2} \mathrm{~s}^{-1}\right)$, respectively. The size of the two metallacycles can also be calculated according to the Stocks-Einstein equation (eqn (1)):

$$
D=K_{\mathrm{b}} T / 6 \pi \eta r_{\mathrm{s}}
$$

Where $K_{\mathrm{b}}$ is the Boltzmann constant, $T$ is the absolute temperature, $\eta$ is the solvent viscosity, and $r_{\mathrm{s}}$ is the hydrodynamic radius. The calculated molecular diameters are about 3.15 and $2.81 \mathrm{~nm}$ for $\mathrm{p}-[3+3]$ and $\mathrm{p}-[2+2]$, respectively.

\section{Self-assembly of anti-parallel conformers and the closed form into hexagonal metallacycles}

Next, we focused on self-assembly of ap-PY with the diplatinum ligand to study the difference between the two conformers in self-assembly based on the successful conformer separation (Scheme 3). Still based on the established method, the large assembled hexagon ap-[6 + 6] can be easily obtained (Scheme $\mathrm{S} 2 \dagger)$. The quantitative formation of hexagon ap-[6+6] was confirmed by multinuclear NMR $\left({ }^{1} \mathrm{H}\right.$ and $\left.{ }^{31} \mathrm{P}\right)$ and ESI-TOF-MS (Fig. 3 and $55 \dagger$ ). The simple ${ }^{1} \mathrm{H}$ NMR pattern of ap- $[6+6]$ showed a similar down-field shift to the parallel conformerbased metallacycles in both pyridine and benzothiophene units due to the loss of electron density upon coordination to the Pt(II) metal center. Meanwhile, the ${ }^{31} \mathrm{P}$ NMR spectrum showed a sharp single peak shifted upfield by 8.00 ppm from initial di-platinum acceptor $\mathbf{1}$, along with a decrease in the coupling of flanking ${ }^{195} \mathrm{Pt}$ satellites $(\Delta J=134.7 \mathrm{~Hz}$, Fig. $3 \mathrm{~b})$, indicative of the discrete and high symmetry structure. The corresponding isotope patterns of ESI-TOF-MS observed were in good agreement with the calculated $\mathrm{m} / \mathrm{z}$ ratios, which further confirmed the formation of larger metallacycles ap- $[6+6]$ by changing the conformer of the unassembled ligand.

Then we further studied the self-assembly of ap-PY with smaller angle $\left(120^{\circ}\right)$ di-Pt(II) acceptor 2 . The discrete smaller cyclotrimer ap-[3 + 3] can also be obtained straightforward. Multinuclear NMR $\left({ }^{1} \mathrm{H}\right.$ and $\left.{ }^{31} \mathrm{P}\right)$ and ESI-MS-TOF evidenced the stoichiometry of formation of ap-[3+3] as well (Fig. 3a, S6 and $\mathrm{S} 7 \dagger)$. Upon self-assembly to form highly symmetrical hexagon ap-[3 + 3], ${ }^{31} \mathrm{P}$ NMR also exhibited a single peak shifted upfield 
a) $\theta=155^{\circ}$

a)

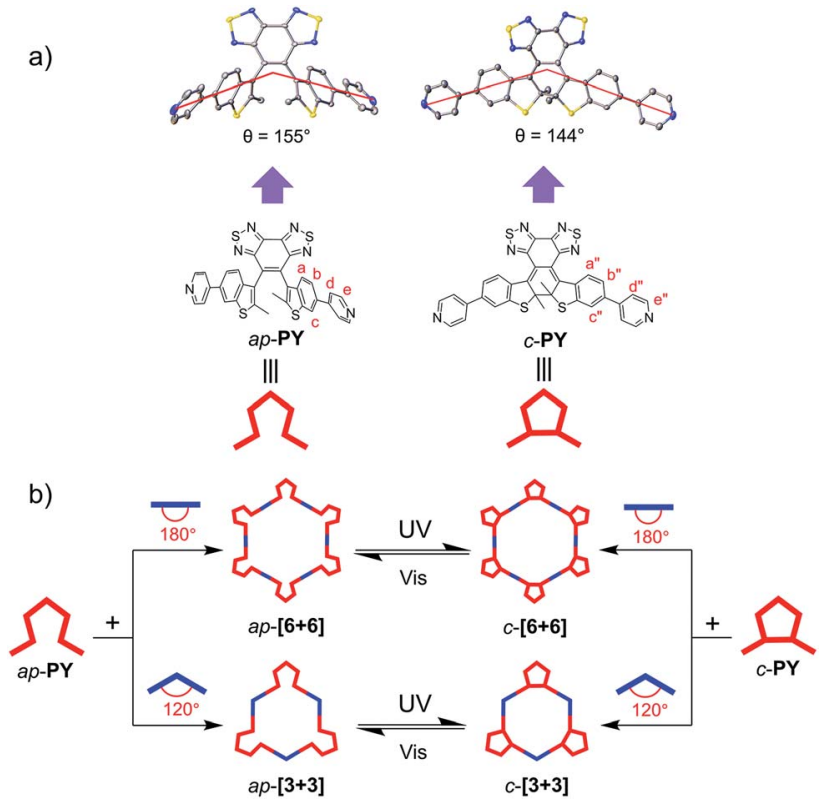

Scheme 3 Graphical representation of self-assembly of ap-PY and CPY with $180^{\circ}$ and $120^{\circ} \mathrm{di}-\mathrm{Pt}(\mathrm{I})$ acceptors 1 and 2, and structural transformation of hexagons with ORTEP representation of X-ray single crystal structures of ap-PY and c-PY drawn with $50 \%$ probability. The red line shows the bending angle of two pyridines.

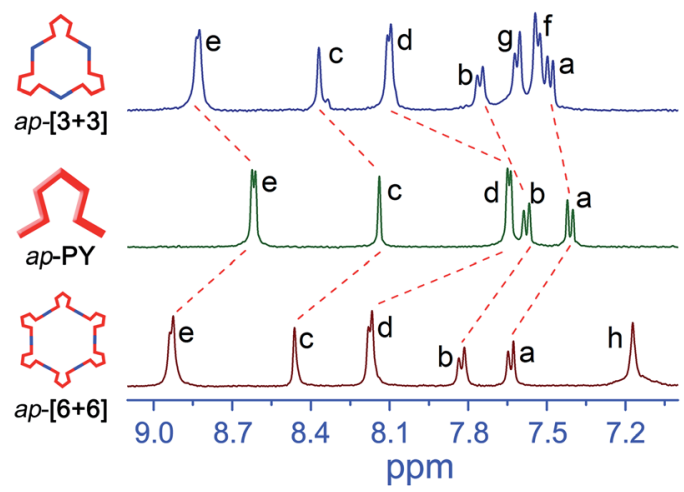

b)

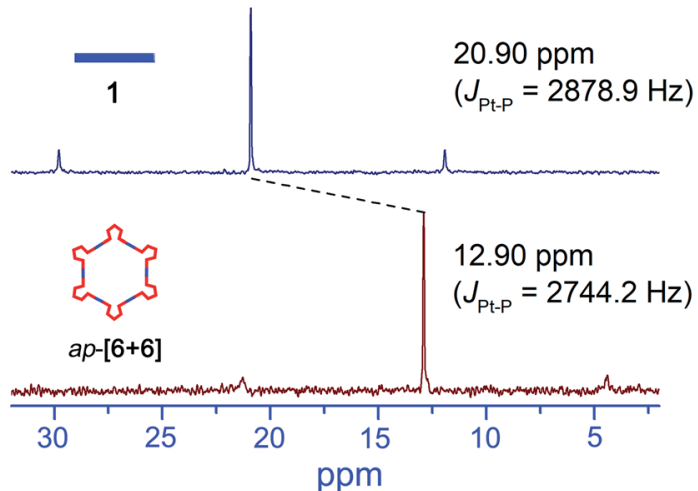

Fig. 3 Characterization of hexagon ap-[3+3] and ap-[6+6]. (a) Partial ${ }^{1} \mathrm{H}$ NMR spectra $(400 \mathrm{MHz}, 293 \mathrm{~K})$ of ap-[3 + 3], ap-PY and ap-[6 +6 ] in a mixture of $\mathrm{CD}_{2} \mathrm{Cl}_{2}$ and acetone- $d_{6}$. (b) ${ }^{31} \mathrm{P}$ NMR spectra (161.9 $\mathrm{MHz}, \mathrm{CD}_{2} \mathrm{Cl}_{2}, 293 \mathrm{~K}$ ) of $180^{\circ}$ di-Pt(॥) acceptor 1 and ap-[3 + 3]. from initial di-platinum acceptor 2 (Fig. S6†). Also in ESI-TOFMS, the peaks at $m / z=1039.51$ and 1336.62 , exactly corresponding to $[\mathrm{M}-5 \mathrm{OTf}]^{5+}$ and $[\mathrm{M}-4 \mathrm{OTf}]^{4+}$, respectively, are in good agreement with the theoretical distribution of the discrete nature of hexagon ap-[3+3] (Fig. S6 and S7 $\dagger$ ).

Regarding the ap-metallacycles, we also performed $2 \mathrm{D}{ }^{1} \mathrm{H}$ DOSY to visualize the size of metallacycles. The observation of a single band of both ap- $[6+6]$ and ap- $[3+3]$ indicated the discrete structure of these two metallacycles. The diffusion coefficients $(D)$ were about $9.12 \times 10^{-11}$ and $1.29 \times 10^{-10} \mathrm{~m}^{2}$ $\mathrm{s}^{-1}$, respectively, in $\mathrm{CD}_{2} \mathrm{Cl}_{2}$, corresponding to objects with diameters of about 10.9 and $7.7 \mathrm{~nm}$ for ap-[6+6] and ap-[3+3], showing the larger size of ap-[6+6] (Fig. 4). Moreover, compared with the p-conformer upon coordination to the same di-Pt(II) acceptor, ap-conformer-based metallacycles possess a larger size and different shapes, suggestive of the different selfassembly behavior between ap- and p-conformers.

Upon changing the photoresponsive ligands and di-Pt(II) acceptors with different bending angles, we obtained a series of metallacycles with different sizes and different shapes. As for p$\mathbf{P Y}$, given that the sterically hindered ethene bridge and benzothiophene unit can block the rotation of side units, the two pyridine units in the parallel conformer p-PY can only extend to the same sides $\left(65^{\circ}\right.$, Scheme 2$)$. This factor limits the bending angles of p-PY. In contrast, the pyridine units in the anti-parallel conformer ap-PY can extend to different sides with larger angle $\left(155^{\circ}\right.$, Scheme 3), thereby forming larger polygon cycles. Meanwhile, it is notable that compared to the parallel conformer, ap-conformer-based metallacycles possess much simpler ${ }^{1} \mathrm{H}$ NMR patterns because of the larger polygon cycles, leading to lower ring tension and reduce the hindered rotation of the Pt-N (pyridine) bond as well as bisbenzo(thiadiazole) ethene bridge between benzothiophene units. ${ }^{41,55}$

Furthermore, hexagon c-[6 + 6] can also be constructed by the closed form donor c-PY through a similar method (Scheme $\mathrm{S} 3 \uparrow) .{ }^{1} \mathrm{H}$ and ${ }^{31} \mathrm{P}$ NMR and ESI-TOF-MS confirmed the quantitative formation of the discrete structure of hexagon c-[6 +6] (Fig. S8-S11 $\dagger$ ). 2D ${ }^{1} \mathrm{H}$ DOSY measurements were also performed for c-[6+6], along with a diameter of about $11.7 \mathrm{~nm}$ fitting to the size of ap-[6+6] (Fig. S12 and S13†).

\section{Conformer-based self-sorting behavior}

Obviously, the self-assembly of ap- and p-conformers with two different $\mathrm{Pt}(\mathrm{II})$ ligands exhibits a series of discrete a)

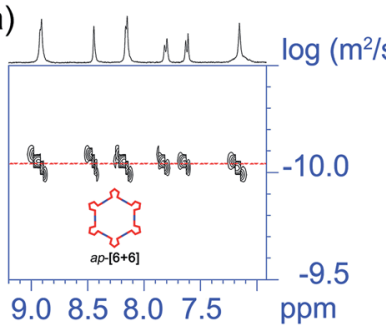

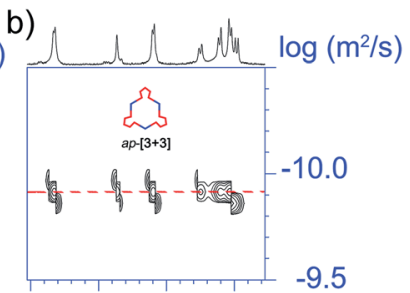

$\begin{array}{llll}8.5 & 8.0 & 7.5 & \mathrm{ppm}\end{array}$
Fig. 4 Partial 2D DOSY spectrum of (a) ap-[6+6] and (b) ap-[3+3], indicating that the calculated molecular diameters are about 10.9 and $7.7 \mathrm{~nm}$ for $\mathrm{ap}-[6+6]$ and $\mathrm{ap}-[3+3]$, respectively. 
supramolecular metallacycles. To further study the conformerdependent self-assembly, we further investigated the behavior of the equimolar ratio mixture of ap-PY and p-PY with a stoichiometric amount of di-Pt(II) acceptor 1 in $\mathrm{CD}_{2} \mathrm{Cl}_{2}$ and DMSO$d_{6}$ solution. Interestingly, the resulting mixtures revealed the completely selective self-assembly of ap-[6+6] and p-[3+3]. In ${ }^{1} \mathrm{H}$ NMR and 2D DOSY spectra (Fig. 5), only the peaks corresponding to hexagon ap-[6 +6] and triangle $\mathrm{p}-[\mathbf{3}+3]$ were observed, with no peaks for other mixed species. ${ }^{31} \mathrm{P}$ NMR can also confirm the self-sorting behavior (Fig. S14 $\dagger$ ). Clearly, it is the mismatched geometrical conformation between ap-PY and p-PY that leads to completely self-sorting behavior. ${ }^{56-58}$

However, compared to ap- and p-conformers, the differences of the bending angles between ap- and c-conformers are smaller. Hence, would it still show the self-sorting behavior of two distinct complexes or the two conformers be self-assembled into a statistical mixture? To solve the issue, the self-sorting behavior between ap- and c-conformers was studied. A mixture of 2.0 eq open form of ap-conformer and 1.0 eq closed form with 3.0 eq Pt salt 1 in $\mathrm{CD}_{2} \mathrm{Cl}_{2}$ and DMSO was prepared. Interestingly, we observed quite clear and similar spectra as shown in Fig. 6 with all peaks corresponding to ap- $[6+6]$ and c$[6+6]$. Moreover, the assembled NMR spectrum showed also the same characteristic as that of the simple mixture of ap-[6+ 6] and $c-[6+6]$ (ratio $=2: 1$, Fig. 6 and S15 $\dagger$ ). It displayed an obvious self-sorting behavior between the open form and the closed form.

Actually, the open form exhibits $155^{\circ}$, and the closed form shows $144^{\circ}$ (Scheme 3). Though the difference is not large, the open form still assembles with an open form to result in an open-form metallacycle, and the closed form only assembles with a closed form to produce a ring-closed metallacycle. Clearly, the assembly is critically dependent upon the specific bending angles of the open-form and closed form. ${ }^{59}$ Moreover, the similar self-sorting behavior can also be found when ap-PY and p-PY or ap-PY and c-PY coordinate with Pt(II) salt 2 (Fig. S16 and $\mathrm{S} 17 \dagger)$. The above self-sorting behavior further demonstrates the conformer-dependent self-assembly behavior.

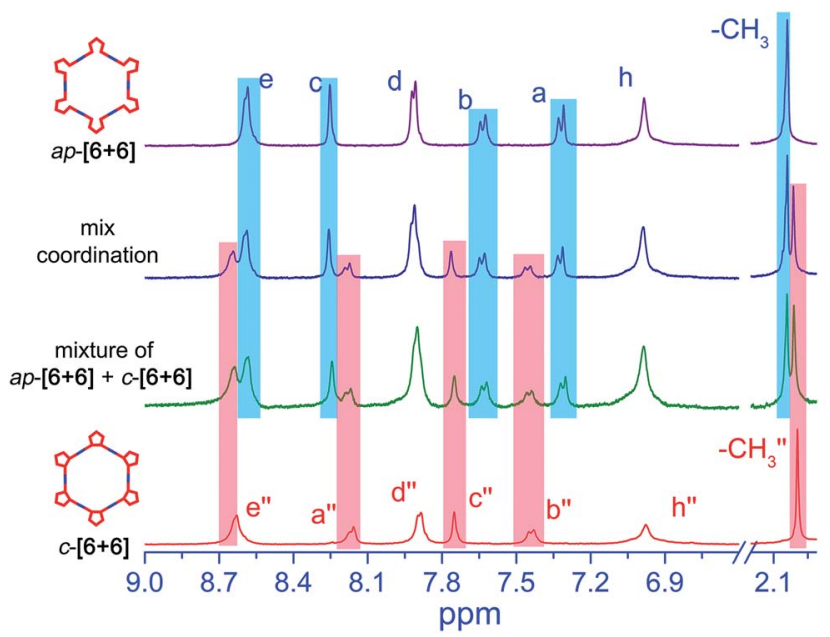

Fig. 6 Characterization of metallacycles assembled by ap-PY and CPY with di-Pt(॥) 1. Comparison of partial ${ }^{1} \mathrm{H}$ NMR $\left(400 \mathrm{MHz}, \mathrm{CD}_{2} \mathrm{Cl}_{2}\right.$ and DMSO- $\left.d_{6}, 293 \mathrm{~K}\right)$ spectra of ap-[6+6], mixed coordination of 2.0 eq ap-PY and 1.0 eq c-PY with 3.0 eq di-Pt(॥) 1 acceptor, mixture of ap-[6 $+6]+c-[6+6]$ (about $2: 1)$, and $c-[6+6]$.

\section{Photo-reversible response of self-assembled metallacycles}

With the supramolecular system in hand, we turn our attention to the photoresponsive performance of ligands and metallacycles. According to the Woodward-Hoffmann rule, for photoresponsive DAE derivatives, only the ap-conformer can undergo photocyclization while p-conformer cannot. That is, the photocyclization quantum yield $\left(\Phi_{\mathrm{O}-\mathrm{c}}\right)$ in conventional DAEs is usually limited to $50 \%$. As expected for the p-conformer, the corresponding p-PY, p- $[2+2]$ and $\mathrm{p}-[3+3]$ didn't show any change both in absorption and emission spectra under UV light irradiation in $\mathrm{CH}_{2} \mathrm{Cl}_{2}$ solution (Fig. S21-S23†). As for apconfiguration, both the ligand and metallacycles show excellent photoresponsive performance (Scheme 1 and Fig. 7a), especially for the photocyclization quantum yield $\left(\Phi_{\mathrm{o}-\mathrm{c}}\right)$ of ap-[6 + 6] and ap-[3 + 3] as high as $61.2 \%$ and $66.8 \%$ (Table 1), respectively.
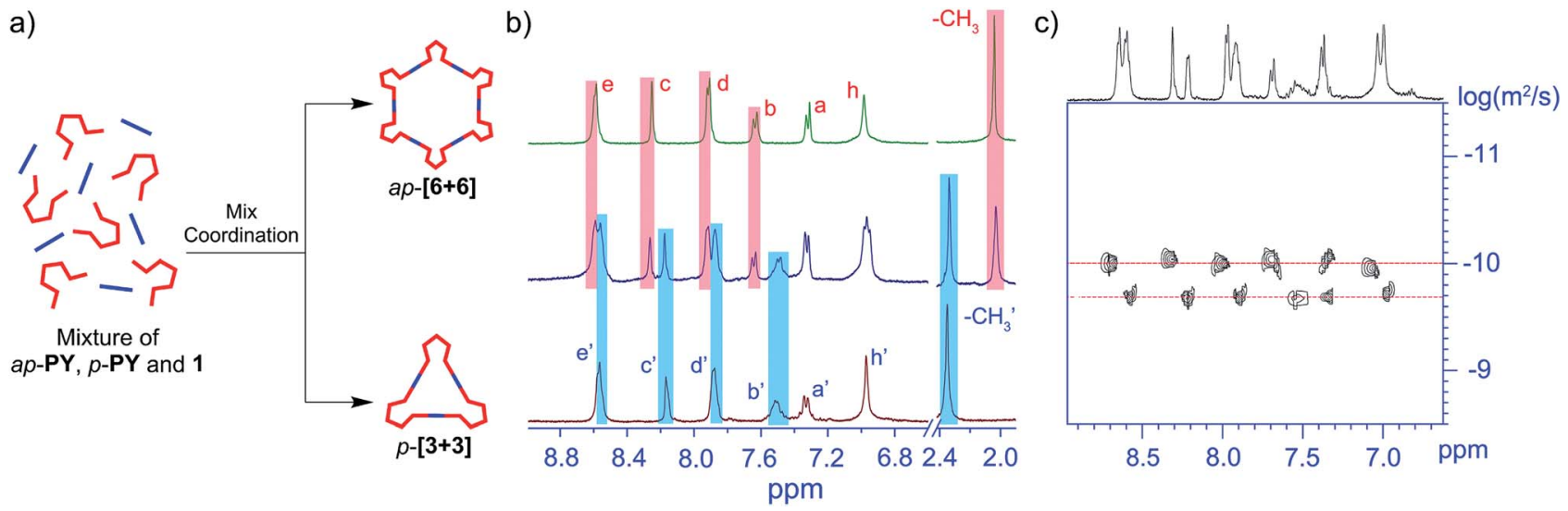

Fig. 5 Mixed self-assembly of ap-PY and p-PY with di-Pt(॥) 1 in $\mathrm{CD}_{2} \mathrm{Cl}_{2}$ and DMSO- $d_{6}$. (a) Graphical representation of mixed self-assembly of apPY and p-PY with di-Pt(I) 1. (b) Partial ${ }^{1} \mathrm{H}$ NMR spectral comparison $(400 \mathrm{MHz}, 293 \mathrm{~K})$ of ap-[6 + 6], p-[3 + 3], and a mixed assembly system. (c) Partial 2D DOSY spectrum of mixed assembly system. 


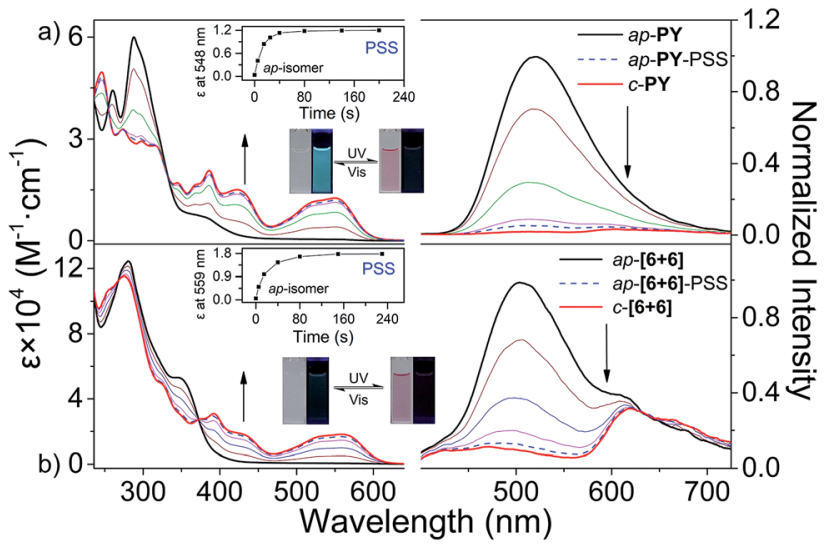

Fig. 7 Light induced spectral change of photoresponsive metallacycles. Absorption and fluorescence spectra of (a) ap-PY and (b) ap-[6 +6 ], respectively, under irradiation with UV light $(\lambda=302 \pm 20 \mathrm{~nm})$ in $\mathrm{CH}_{2} \mathrm{Cl}_{2}$. Excitation for fluorescence is set at each isosbestic point. The inset images show the color and emission changes triggered by UV ( $\lambda$ $=302 \pm 20 \mathrm{~nm})$ and $\mathrm{Vis}(\lambda>480 \mathrm{~nm})$ light and the spectral changes of extinction coefficient.

Here, we mainly focus on the photoresponsive properties of self-assembly systems. Different from traditional selfassembly systems, these metallacycles (ap-[6 + 6] and ap- $[3+$ 3]) are a series of photo-active self-assembly systems which can transform reversibly between open- and closed-forms triggered by light (Scheme 1b). Similar to free ligand ap-PY, ap-[6 + 6] showed an intense absorption band at $280 \mathrm{~nm}$ corresponding to the bisbenzo(thiadiazole) ethene bridge, along with a medium shoulder peak at about 340-430 $\mathrm{nm}$. Upon UV light $(302 \pm 20 \mathrm{~nm})$ irradiation of ap- $[6+6]$ in $\mathrm{CH}_{2} \mathrm{Cl}_{2}$ solution, the color turned red, similar to ap-PY, with the appearance of three new peaks at 559, 426 and $392 \mathrm{~nm}$, showing a clear isosbestic point without a redshift during the phototransformation process. Here the phenomenon arose from the typical photocyclization in formation of the corresponding closed metallacycles (c-[6 + 6], Fig. 7b). Compared with c-PY, the three absorption peaks of the photoresponsive $c-[6+6]$ exhibited a red-shift by 11,5 and $8 \mathrm{~nm}$, indicative of an extended $\pi$ conjugation upon coordination of ap-PY with acceptor 1. Moreover, the assembled metallacycles in ap-, pand c-forms exhibited higher absorption coefficients based on each photochromic unit (Table 1 and Fig. S28†). The similar coordination metallacycle ap-[3 + 3] also exhibited excellent photoswitchable performance (Fig. S24†). Notably, the conversion yield for both different ring size hexagons in ap-[6 $+6]$ and ap-[3+3] is more than $90 \%$, which is comparable to that for the photochromic ligand ap-PY (Table 1). It is indicative that, upon coordination-induced self-assembly with the $\mathrm{Pt}$ (II) acceptor, the supramolecular hexagons can still keep good photo-switchable characteristics.

Meanwhile, as powerful tools, ${ }^{1} \mathrm{H}$ and ${ }^{31} \mathrm{P}$ NMR spectroscopy techniques can be used to monitor the UV light-induced transformation process from ap- $[6+6]$ to $c-[6+6]$. Upon irradiation with $302 \pm 20 \mathrm{~nm}$ light, the ${ }^{1} \mathrm{H}$ NMR spectra (Fig. S30 $\dagger$ ) during the light-induced transformation from ap-[6 + 6] to c- $[6+$ 6] showed distinct changes, especially in $H_{\mathrm{a}}$ shifting from 7.51 to $8.20 \mathrm{ppm}$, attributed to the formation of intramolecular hydrogen bonds between $H_{\mathrm{a}}$ and the $\mathrm{N}$ atom of thiadiazole. We observed clear and simple ${ }^{1} \mathrm{H}$ NMR spectra illustrating the maintenance of the high symmetry structures during the photoisomerization process. All peaks can be ascribed to ap-[6+6] and $c-[6+6]$, whereas no other peaks belonging to the intermediate states could be found.

Additionally, the fluorescence of ap-metallacycles can also be photo-modulated. For instance, similar to photoresponsive ligand ap-PY (Fig. 7a), metallacycle ap-[6 + 6] showed a weak green fluorescence emission band at 400$725 \mathrm{~nm}$ upon excitation at $375 \mathrm{~nm}$ along with an unexpected shoulder peak at about $615 \mathrm{~nm}$ (Fig. 7b). Subsequently, upon UV light irradiation at $302 \pm 20 \mathrm{~nm}$, the emission of open metallacycle ap-[6+6] converted into closed c-[6+6] became seriously quenched in fluorescence by $96.5 \%$ at the

Table 1 Spectroscopic data of the ligands and self-assembly system in $\mathrm{CH}_{2} \mathrm{Cl}_{2}$ solution

\begin{tabular}{|c|c|c|c|c|c|c|c|c|c|c|}
\hline Compounds & $\begin{array}{l}\lambda_{\mathrm{abs}, \max ^{a}}(\mathrm{~nm}) \\
{\left[\varepsilon_{\mathrm{p}}^{b} / \varepsilon_{\mathrm{m}}\right.} \\
\left.\left(10^{3} \mathrm{M}^{-1} \mathrm{~cm}^{-1}\right)\right]\end{array}$ & $\mathrm{CR}_{\mathrm{O}-\mathrm{c}}{ }^{c}[\%]$ & $\mathrm{CR}_{\mathrm{c}-\mathrm{o}}{ }^{c}[\%]$ & $\Phi_{\mathrm{o}-\mathrm{c}}{ }^{d}[\%]$ & $\Phi_{\mathrm{c}-\mathrm{o}}^{d}[\%]$ & $\Phi_{\mathrm{F}}^{e}(\%)$ & $\begin{array}{l}\lambda_{\mathrm{em} 1} \\
{[\mathrm{~nm}]}\end{array}$ & $\tau_{1}[\mathrm{~ns}]$ & $\begin{array}{l}\lambda_{\mathrm{em} 2} \\
{[\mathrm{~nm}]}\end{array}$ & $\tau_{2}[\mathrm{~ns}]$ \\
\hline c-PY & $549[12.6]$ & - & $>99$ & - & 4.6 & - & - & - & - & - \\
\hline p-PY & $285[66.4]$ & - & - & - & - & 0.33 & 494 & 0.26 & - & - \\
\hline ap- $[6+6]$ & $280[124.5 / 747.0]$ & 90 & - & 61.2 & - & 0.10 & 503 & 1.51 & 615 & 1.74 \\
\hline ap- $[3+3]$ & $283[96.3 / 289.6]$ & 93 & - & 66.8 & - & 0.14 & 481 & 0.41 & 618 & 1.50 \\
\hline$c-[3+3]$ & $562[17.2 / 51.6]$ & - & $>99$ & - & 6.3 & - & - & - & - & - \\
\hline $\mathrm{p}-[2+2]$ & $283[68.9 / 137.8]$ & - & - & - & - & 0.8 & 467 & 1.02 & 578 & 2.05 \\
\hline
\end{tabular}

${ }^{a}$ Typical absorption maxima of the ring-open isomer in the UV region and the ring-closed isomer in the visible region, respectively. ${ }^{b} \varepsilon_{\mathrm{p}}$ represents the molar extinction coefficient of metallacycles calculated by the concentration of the photoresponse unit and $\varepsilon_{\mathrm{m}}$ represents the molar extinction coefficient of metallacycles calculated by the concentration of metallacycles. ${ }^{c}$ Conversion ratio from open to closed isomers (irradiation at $\lambda=302$ $\pm 20 \mathrm{~nm}$ ) and ring-open isomers (under visible light irradiation, $\lambda>480 \mathrm{~nm}$ ), calculated from absorption spectra. ${ }^{d}$ Quantum yields of photocyclization $\left(\Phi_{\mathrm{o}-\mathrm{c}}\right)$ at $313 \mathrm{~nm}$ and cycloreversion $\left(\Phi_{\mathrm{c}-\mathrm{o}}\right)$ at $517 \mathrm{~nm} .{ }^{e}$ Fluorescence quantum yield $\left(\Phi_{\mathrm{F}}\right)$. 
photostationary state (PSS). The unexpected fluorescence shoulder peak has been reported previously in a $[3+3]$ system. ${ }^{\mathbf{4 4}}$ Here we further study the origination of the shoulder peak in ap-[6+6]. The fluorescence lifetime monitored by the additional peak at around $600 \mathrm{~nm}$ was in the range of nanoseconds, indicative of the typical fluorescence characteristic, not phosphorescence (Table 1 and Fig. S31$\mathrm{S} 34 \dagger)$. The reference mixture with the di-platinum acceptor in $1: 1$ ratio can further confirm that the additional fluorescence still might be attributed to the self-assembled formation of metallacycles (Fig. S35†).

In this way, we can achieve the supramolecular photoswitched reversibility with the implanted photoresponsive unit, which is in stark contrast with the traditional "inactive" coordination-induced metallacycles. For both ap-[3+3] and ap- $[6+6]$, irradiation with visible light $(\lambda>480 \mathrm{~nm})$ can bleach the red solution and regenerate the open ring isomers. As a matter of fact, the alternating irradiation with UV $(302 \pm 20$ $\mathrm{nm})$ and visible light $(\lambda>480 \mathrm{~nm})$ toggled the photoswitch repeatedly between the open and closed hexagon metallacycles with remarkable fatigue resistance (Fig. S36†). For both different ring size hexagons, the quantum yields of photocyclization and cycloreversion in ap- $[6+6]$ and ap- $[3+3]$ were almost the same (Table 1). Compared to the photochromic ligand ap-PY, the quantum yields decreased slightly upon coordination, which can be attributed to the metallacycle ring tension to some restriction in the rotation of benzothiophene units. ${ }^{42,60,61}$

\section{Conclusions}

In summary, we have for the first time constructed a unique series of conformer-dependent photoactive metallacycles, especially focusing on the characterization and comparison of self-assembly behavior in the separated photoresponsive ligand conformers with different di-platinum(II) acceptors. The scaffold sizes and shapes of photoswitchable metallacycles can be precisely controlled by photochromic parallel or anti-parallel conformers via coordination-driven self-assembly. Along with the specific conformer-based self-sorting behavior, the apconformer and closed form provide larger bending angles upon coordination with di-platinum(II) acceptors into hexagon $[6+6]$ or $[3+3]$ while p-conformer can only form smaller polygon cycles with triangle or rhombus cycles. Furthermore, taking advantage of the separated ap-conformer ligand, it possesses excellent photoresponsive performance with a relatively high conversion ratio and well-controlled reversible structure transformation, especially for realizing the photocyclization quantum yield $\left(\Phi_{\mathrm{o}-\mathrm{c}}\right)$ of ap- $[6+6]$ and ap- $[3+3]$ as high as $61.2 \%$ and $66.8 \%$, respectively, making a breakthrough to the general limitation to $50 \%$ in conventional diarylethene systems. We can achieve the supramolecular photo-switchable reversibility with the implanted photoresponsive unit, which is in stark contrast with the traditional "inactive" coordinationinduced metallacycles. This work would be a good model to develop soft smart materials for use in probing, catalysis and guest encapsulation systems.

\section{Conflicts of interest}

There are no conflicts to declare.

\section{Acknowledgements}

W.-H. Z acknowledges financial support from the NSFC for Science Center Program (21788102), Creative Research Groups (21421004) and Key Project (21636002), NSFC/China, the National key Research and Development Program (2016YFA0200300), the Shanghai Municipal Science and Technology Major Project (2018SHZDZX03), and the Program of Introducing Talents of Discipline to Universities (B16017). H.-B. $\mathrm{Y}$ acknowledges the financial support of NSFC/China (No. 21625202), the 973 Program (No. 2015CB856600), STCSM (No. 16XD1401000), and the Program for Changjiang Scholars and Innovative Research Team in University.

\section{Notes and references}

1 P. J. Stang and B. Olenyuk, Acc. Chem. Res., 1997, 30, 502.

2 T. R. Cook and P. J. Stang, Chem. Rev., 2015, 115, 7001.

3 J.-M. Lehn, Chem. Soc. Rev., 2007, 36, 151.

4 M. Fujita, M. Tominaga, A. Hori and B. Therrien, Acc. Chem. Res., 2005, 38, 369.

5 A. J. McConnell, C. S. Wood, P. P. Neelakandan and J. R. Nitschke, Chem. Rev., 2015, 115, 7729.

6 G. Charalambidis, E. Georgilis, M. K. Panda, C. E. Anson, A. K. Powell, S. Doyle, D. Moss, T. Jochum, P. N. Horton, S. J. Coles, M. Linares, D. Beljonne, J. V. Naubron, J. Conradt, H. Kalt, A. Mitraki, A. G. Coutsolelos and T. S. Balaban, Nat. Commun., 2016, 7, 12657.

7 X. Yan, T. R. Cook, P. Wang, F. Huang and P. J. Stang, Nat. Chem., 2015, 7, 342.

8 Y. Ueda, H. Ito, D. Fujita and M. Fujita, J. Am. Chem. Soc., 2017, 139, 6090.

9 K. Wu, K. Li, Y. J. Hou, M. Pan, L. Y. Zhang, L. Chen and C. Y. Su, Nat. Commun., 2016, 7, 10487.

10 C. M. Hong, R. G. Bergman, K. N. Raymond and F. D. Toste, Acc. Chem. Res., 2018, 51, 2447.

11 A. I. d'Aquino, Z. S. Kean and C. A. Mirkin, Chem. Mater., 2017, 29, 10284.

12 L. J. Chen, S. Chen, Y. Qin, L. Xu, G. Q. Yin, J. L. Zhu, F. F. Zhu, W. Zheng, X. Li and H. B. Yang, J. Am. Chem. Soc., 2018, 140, 5049.

13 W. Zheng, W. Wang, S. T. Jiang, G. Yang, Z. Li, X. Q. Wang, G. Q. Yin, Y. Zhang, H. Tan, X. Li, H. Ding, G. Chen and H. B. Yang, J. Am. Chem. Soc., 2018, 141, 583.

14 W. Wang, Y. X. Wang and H. B. Yang, Chem. Soc. Rev., 2016, 45, 2656.

15 S. Das, P. Ranjan, P. S. Maiti, G. Singh, G. Leitus and R. Klajn, Adv. Mater., 2013, 25, 422.

16 H. F. Cheng, A. I. d'Aquino, J. Barroso-Flores and C. A. Mirkin, J. Am. Chem. Soc., 2018, 140, 14590.

17 J. Zhu, Y. Kim, H. Lin, S. Wang and C. A. Mirkin, J. Am. Chem. Soc., 2018, 140, 5061. 
18 J. Zhou, M. Chen and G. Diao, ACS Appl. Mater. Interfaces, 2014, 6, 18538.

19 N. Kishi, M. Akita, M. Kamiya, S. Hayashi, H.-F. Hsu and M. Yoshizawa, J. Am. Chem. Soc., 2013, 135, 12976.

20 W. M. Bloch, J. J. Holstein, W. Hiller and G. H. Clever, Angew. Chem., Int. Ed., 2017, 56, 8285.

21 S. Oldknow, D. R. Martir, V. E. Pritchard, M. A. Blitz, C. W. G. Fishwick, E. Zysman-Colman and M. J. Hardie, Chem. Sci., 2018, 9, 8150.

22 S. C. Wei, M. Pan, Y. Z. Fan, H. Liu, J. Zhang and C. Y. Su, Chem.-Eur. J., 2015, 21, 7418.

23 S. Chen, L. J. Chen, H. B. Yang, H. Tian and W. Zhu, J. Am. Chem. Soc., 2012, 134, 13596.

$24 \mathrm{H}$. Yotsuji, K. Higashiguchi, R. Sato, Y. Shigeta and K. Matsuda, Chem.-Eur. J., 2017, 23, 15059.

25 P. K. Kundu, S. Das, J. Ahrens and R. Klajn, Nanoscale, 2016, 8, 19280.

26 Y. Han, C. Gao and X. He, Sci. China: Chem., 2011, 55, 604.

27 K. K. Kartha, N. K. Allampally, A. T. Politi, D. D. Prabhu, H. Ouchi, R. Q. Albuquerque, S. Yagai and G. Fernández, Chem. Sci., 2019, 10, 752.

28 D. Zhang, Y. Nie, M. L. Saha, Z. He, L. Jiang, Z. Zhou and P. J. Stang, Inorg. Chem., 2015, 54, 11807.

29 M. Irie, Chem. Rev., 2000, 100, 1685.

30 M. Irie, T. Fukaminato, K. Matsuda and S. Kobatake, Chem. Rev., 2014, 114, 12174.

31 J. Su, T. Fukaminato, J. P. Placial, T. Onodera, R. Suzuki, H. Oikawa, A. Brosseau, F. Brisset, R. Pansu, K. Nakatani and R. Métivier, Angew. Chem., Int. Ed., 2016, 55, 3662.

32 C.-W. Hsu, C. Sauvée, H. Sundén and J. Andréasson, Chem. Sci., 2018, 9, 8019.

33 M. Fukagawa, I. Kawamura, T. Ubukata and Y. Yokoyama, Chem.-Eur. J., 2013, 19, 9434.

34 Y. Cai, Y. Gao, Q. Luo, M. Li, J. Zhang, H. Tian and W.-H. Zhu, Adv. Opt. Mater., 2016, 4, 1410.

35 N. M.-W. Wu, M. Ng and V. W.-W. Yam, Angew. Chem., Int. Ed., 2019, 58, 3027.

36 E. Siemes, O. Nevskyi, D. Sysoiev, S. K. Turnhoff, A. Oppermann, T. Huhn, W. Richtering and D. Wöll, Angew. Chem., Int. Ed., 2018, 57, 12280.

37 Y. Zheng, H. Sato, P. Wu, H. J. Jeon, R. Matsuda and S. Kitagawa, Nat. Commun., 2017, 8, 100.

38 F. Eisenreich, M. Kathan, A. Dallmann, S. P. Ihrig, T. Schwaar, B. M. Schmidt and S. Hecht, Nat. Catalysis, 2018, 1, 516.

39 X. Hu, M. E. McFadden, R. W. Barber and M. J. Robb, J. Am. Chem. Soc., 2018, 140, 14073.
40 M. Han, Y. Luo, B. Damaschke, L. Gómez, X. Ribas, A. Jose, P. Peretzki, M. Seibt and G. H. Clever, Angew. Chem., Int. Ed., 2016, 55, 445.

41 M. Han, R. Michel, B. He, Y. S. Chen, D. Stalke, M. John and G. H. Clever, Angew. Chem., Int. Ed., 2013, 52, 1319.

42 W. Li, C. Jiao, X. Li, Y. Xie, K. Nakatani, H. Tian and W. Zhu, Angew. Chem., Int. Ed., 2014, 53, 4603.

43 W. Li, X. Li, Y. Xie, Y. Wu, M. Li, X. Y. Wu, W. H. Zhu and H. Tian, Sci. Rep., 2015, 5, 9186.

44 M. Li, L.-J. Chen, Y. Cai, Q. Luo, W. Li, H.-B. Yang, H. Tian and W.-H. Zhu, Chem, 2019, 5, 634.

45 J. Yoon and A. P. de Silva, Angew. Chem., Int. Ed., 2015, 54, 9754.

46 K. Morinaka, T. Ubukata and Y. Yokoyama, Org. Lett., 2009, 11, 3890.

47 T. Nakashima, R. Fujii and T. Kawai, Chem.-Eur. J., 2011, 17, 10951.

48 M. Kamrul Hossain, M. Takeshita and T. Yamato, Eur. J. Org. Chem., 2005, 2005, 2771.

49 T. Yamada, K. Muto, S. Kobatake and M. Irie, J. Org. Chem., 2001, 66, 6164.

50 C. S. Pecinovsky, E. S. Hatakeyama and D. L. Gin, Adv. Mater., 2008, 20, 174.

51 Y.-F. Han, G.-X. Jin and F. E. Hahn, J. Am. Chem. Soc., 2013, 135, 9263.

52 S. R. Seidel and P. J. Stang, Acc. Chem. Res., 2002, 35, 972.

53 X. Yan, H. Wang, C. E. Hauke, T. R. Cook, M. Wang, M. L. Saha, Z. Zhou, M. Zhang, X. Li, F. Huang and P. J. Stang, J. Am. Chem. Soc., 2015, 137, 15276.

54 G. Tárkányi, H. Jude, G. Pálinkás and P. J. Stang, Org. Lett., 2005, 7, 4971.

55 H.-B. Yang, N. Das, F. Huang, A. M. Hawkridge, D. D. Díaz, A. M. Arif, M. G. Finn, D. C. Muddiman and P. J. Stang, J. Org. Chem., 2006, 71, 6644.

56 M. M. Safont-Sempere, G. Fernández and F. Würthner, Chem. Rev., 2011, 111, 5784.

57 C. Gütz, R. Hovorka, C. Klein, Q. Q. Jiang, C. Bannwarth, M. Engeser, C. Schmuck, W. Assenmacher, W. Mader, F. Topić, K. Rissanen, S. Grimme and A. Lützen, Angew. Chem., Int. Ed., 2014, 53, 1693.

58 F. J. Rizzuto, M. Kieffer and J. R. Nitschke, Chem. Sci., 2018, 9, 1925.

59 M. Wang, C. Wang, X. Q. Hao, X. Li, T. J. Vaughn, Y. Y. Zhang, Y. Yu, Z. Y. Li, M. P. Song, H. B. Yang and X. Li, J. Am. Chem. Soc., 2014, 136, 10499.

60 S. Aloïse, M. Sliwa, Z. Pawlowska, J. Réhault, J. Dubois, O. Poizat, G. Buntinx, A. Perrier, F. Maurel, S. Yamaguchi and M. Takeshita, J. Am. Chem. Soc., 2010, 132, 7379.

61 M. Walko and B. L. Feringa, Chem. Commun., 2007, 43, 1745. 